\title{
93 COMPUTATIONAL BIOLOGY AND TISSUE-BASED APPROACHES TO INFORM INDICATION SELECTION FOR A NOVEL AHR INHIBITOR
}

Marta Sanchez-Martin*, Lei Wang, Jeffrey Ecsedy, Karen Mcgovern, Michelle Zhang. Ikena Oncology, Boston, MA, USA

Background Aryl Hydrocarbon Receptor (AHR) is a ligandactivated transcription factor that regulates the activities of multiple innate and adaptive immune cell types. Multiple ligands such as kynurenine bind to AHR driving its nuclear translocation and transcriptional activation, leading to an immunosuppressive tumor microenvironment. ${ }^{1}{ }^{2}$ AHR activation is implicated in tumor development in multiple cancer types. In addition, high levels of serum kynurenine are associated with resistance to checkpoint inhibitors. ${ }^{3}$ To overcome AHR-mediated immunosuppression in cancers, we developed a selective oral AHR inhibitor IK-175 and took a combined computational and tissue-based approach to select cancer indications for its clinical development.

Methods The aim of this work is to identify tumor indications dependent on AHR signaling and design patient selection strategies based on a proprietary transcriptional signature, mRNA and protein detection assays to evaluate AHR pathway activation in tumors.

Results Genomic profiling of solid and hematological cancers from TCGA and Project GENIE databases identified bladder and esophageal tumors among others, as frequently harboring AHR gene amplifications.A proprietary gene signature of AHR activation was developed integrating literature, pathway analysis, RNAseq and nanostring data from PBMC, T-cells and cell lines upon AHR inhibition. Transcriptional analysis of the TCGA data using this signature demonstrated bladder cancer has the highest expressions of AHR and AHR signature genes, suggesting increased pathway activity in bladder cancer relative to other cancer types. Increased AHR signature gene expression was associated with worse overall survival in the TCGA bladder cancer cohort. Furthermore, RNAscope analysis of a tissue microarray containing 10 different tumor types revealed bladder cancer had one of the highest AHR transcript expression in the tumor compartment.Finally, nuclear localization of AHR protein was assessed as an indicator of pathway activation through the development of a novel IHC method. Extensive TMA screening of AHR protein in 15 different indications demonstrated bladder cancer as the tumor type with the highest prevalence of AHR nuclear expression.

Conclusions In summary, we demonstrated high prevalence of nuclear AHR protein expression, AHR gene amplification and target gene expression in bladder cancer, suggesting aberrant AHR activation may play an important role in the progression of this tumor type. This study provides rationale for therapeutic targeting of AHR in bladder cancer patients. Ikena is currently evaluating the anti-tumor activity of IK-175 as a single agent and in combination with nivolumab in bladder cancer in a Phase 1a/1b clinical study (NCT04200963).

\section{REFERENCES}

1. Quintana FJ, Sherr DH. Aryl hydrocarbon receptor control of adaptive immunity. Pharmacol Rev 2013 Aug 1;65(4):1148-61.

2. Murray IA, Patterson AD, Perdew GH. Aryl hydrocarbon receptor ligands in cancer: friend and foe. Nat Rev Cancer 2014 Dec:14(12):801-14.

3. Li, Haoxin et al. 'Metabolomic adaptations and correlates of survival to immune checkpoint blockade.' Nature Communications 2019 Sep 25;10:1-4346.

http://dx.doi.org/10.1136/jitc-2021-SITC2021.093 